\title{
Protocatechuic Acid Levels Discriminate Between Organic and Conventional Wheat from Denmark
}

\author{
Yannick Weesepoel ${ }^{a}$, Samuel Heenan ${ }^{\mathrm{a}}$, Rita Boerrigter-Eenling ${ }^{\mathrm{a}}$, Tjerk Venderink ${ }^{\mathrm{a}}$, Marco Blokland ${ }^{\mathrm{a}}$, \\ and Saskia van Ruth*ab
}

\begin{abstract}
Organic wheat retails at higher market prices than the conventionally grown counterparts. In view of fair competition and sustainable consumer confidence, the organic nature of organic wheat needs to be assured. Amongst other controls this requires analytical tests based on discriminating traits. In this paper, phenolic acids were examined by liquid chromatography analysis as biomarkers for discriminating between the two groups by means of a controlled grown full factorial design Danish wheat sample set. By combining baseline and retention-time correction pre-treatments and principal component analysis, discrimination between organic and conventional produce was found to be expressed in the first principal component (93\%), whilst the second principal component accounted for the production year (4\%). Upon examination of the loadings plot, a single chromatographic peak was found to account for a large part in the discrimination between the two wheat production systems. This was further underpinned by statistically significant differences found in concentrations between the organic and conventional production systems of this phenolic acid (ANOVA, P $<0.05$ ). The phenolic acid was tentatively identified as protocatechuic acid by negative mode mass spectrometry. The results obtained implied that protocatechuic acid may serve as a single marker for discrimination between organic and conventional produced wheat.
\end{abstract}

Keywords: Cereals · Food adulteration · Food fraud · Liquid chromatography · Phenolic acids · Wheat

\section{Introduction}

A cereal is generally defined as a grass grown for its small, edible seed..$^{[1]}$ Cereals are an important carbohydrate source in the human diet. Maize (corn), rice (paddy), wheat, barley and sorghum are the top five cereals in the world when ranked on the basis of production tonnage. ${ }^{[2]}$ In conjunction with the large world-wide cereal consumption, the demand for organically produced foods increased considerably in the last decades. As a consequence in some countries, the market share for organic produce has become a significant percentage of the total market. Organic products tend to retail at a higher price than their conventionally grown/produced counterparts, mainly because of lower yield and certification

\footnotetext{
${ }^{\star}$ Correspondence: Prof. S. Van Ruth ${ }^{\text {ab }}$ E-mail: saskia.vanruth@wur.nl

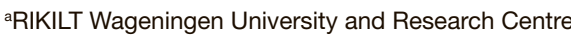
P.O. box 230, 6700 AE Wageningen, The Netherlands ${ }^{b}$ Food Quality and Design Group

Wageningen University

P.O. box 17, 6700 AA Wageningen, The Netherlands
}

costs. This premium price and the increasing demand make organic food products susceptible to mix-up.

Organic production has to comply with specific legislation in the EU (e.g. Regulation (EC) No. 889/2008).[3] According to this legislation, production of organic plant products is severely restricted in the use of synthetic fertilizers, pesticides and insecticides. Organic production is based on i) frequent crop rotations and extensive soil tillage, ii) the use of compost or organic waste rather than chemical-synthetic pesticides and readily soluble mineral fertilizers and iii) biological pest control. ${ }^{[4]}$ It is expected that these distinctive conditions impact on the phytochemical composition of the plants.

Plant material, including cereals, consists of a complex array of phytochemicals that are highly influenced by cultivar and growing/environmental conditions. For instance the phytochemical content and composition is influenced by the availability of sunlight, water and temperature conditions as well as by the availability of nutrients in the soil. Considerable knowledge exists on the levels of phytochemical compounds in various cereals. ${ }^{[5,6]}$ For example, phytochemicals such as the small phenolic acids feruli, $p$-coumaric, syringic, vanillic and caffeic acids were found to be highly variable amongst wheat varieties. Furthermore, these phenolic acids occur in both 'free' and 'bound' forms, adding an extra dimension of complexity for characterization of the wheat varieties. ${ }^{[7]}$ In combination with a relatively straightforward extraction procedure and liquid chromatography analysis, the phenolic acids seem to be an attractive and potential group of biomarkers for discrimination between organic and conventional cereals.

The goal of this study is therefore to examine the levels of phenolics in sets of organic and conventional Danish wheat by liquid chromatography (LC) in combination with statistics considering also the influence of geographical location and harvesting year. This work is part of the EU project 'AuthenticFood'.

\section{Materials and Methods}

\subsection{Materials}

\subsubsection{Chemicals}

Methanol (99.98\%), $n$-hexane (99.95\%) and acetonitrile (99.97\%) were obtained from Actu-All Chemicals (Oss, The Netherlands). Sodium acetate $(99.0 \%)$, sodium chloride (99.99\%) sodium hydroxide $(99.0 \%)$, ethyl acetate $(99.8 \%)$ and hydrochloric acid (37\%) were obtained from Merck Millipore (Darmstadt, Germany). Ultra-pure water was produced by a Millipore Advantage machine (Milllipore, Merck, Germany). 


\subsubsection{Wheat Samples}

A sample set of thirty-six wheat samples (Triticum aestivum L. cv. Tommi) were supplied by partners of the EU Core II Organic project 'AuthenticFood'. The set comprised samples of (a) two variations of organic production (fertilization with animal manure (Organic A), and with cover crop (Organic B)) and conventional production, (b) three geographical locations in Denmark, and (c) two harvesting years (Table 1) as described by Laursen and co-workers. ${ }^{[8]}$ The outline resulted in a full factorial $3 \times 3 \times 2$ design, with each category a duplicate sample batch $(5 \mathrm{~kg}$ per sample obtained by stepwise mass reduction) and for each batch again duplicate sample analyses.

\subsection{Methods}

\subsubsection{Sample Preprocessing and Extraction}

Wheat samples $(10 \mathrm{~g})$ were ground to a fine flour using a blender (Waring Laboratory Science, Winsted, CT, USA). Subsequently, $0.5 \mathrm{~g}$ flour was extracted with $25 \mathrm{~mL} 70 \%(\mathrm{v} / \mathrm{v})$ chilled methanol and mixed head-over-head for $10 \mathrm{~min}$. After centrifugation at approximately $783 \mathrm{~g}$, the supernatant was collected and the extraction procedure was repeated. The two supernatants were pooled and evaporated to dryness at $45^{\circ} \mathrm{C}$ under a stream of $\mathrm{N}_{2}$. Bound phenolics were extracted by digestion of the remaining residue by addition of $10 \mathrm{~mL} 4 \mathrm{M}$ sodium hydroxide solution. Samples were incubated for $1 \mathrm{~h}$ at $60{ }^{\circ} \mathrm{C}$ with a $\mathrm{N}_{2}$ flushed head-space. After neutralization with approximately $10 \mathrm{M}$ hydrochloric acid, samples were centrifuged at approximately $783 \mathrm{~g}$. The obtained supernatant was extracted three times with aliquots of $10 \mathrm{~mL}$ of $n$-hexane to remove interfering lipids. The hexane fractions were discarded. Subsequently, the supernatant was extracted 5 times with aliquots of $10 \mathrm{~mL}$ ethyl acetate. Ethyl acetate fractions were collected and evaporated to dryness at $45{ }^{\circ} \mathrm{C}$ with $\mathrm{N}_{2}$ head-space flush. Prior to LC injection, dried extracts were dissolved in $1 \mathrm{~mL}$ ultrapure water (approximately $1 \mathrm{mg} \mathrm{mL}^{-1}$ ) and filtered through a $0.45 \mu \mathrm{m}$ PTFE filter (Pall, Acrodisc CR, Anotop, Whatman International).

\subsubsection{LC-Diode Array Detection analysis ( $L C-D A D)$}

Chromatographic separation was performed on an Agilent HPLC 1100 series (Agilent Technologies, Amstelveen, The Netherlands) equipped with a Zorbax 300 Extend-C18 $4.6 \times 250 \mathrm{~mm} 5 \mu \mathrm{m}$ column (Agilent), LiChrosorb C18 $4.6 \times 7.5 \mathrm{~mm}$ $5 \mu \mathrm{m}$ precolumn (Agilent) and in-line diode array detector (Agilent). Eluents

Table 1. Sample overview

\begin{tabular}{|l|l|c|c|}
\hline Production system & Location & Harvesting year & Number of batches \\
\hline $\begin{array}{l}\text { Conventional } \\
\text { Organic A }\end{array}$ & Flakkebjerg & 2007 & 2 \\
\hline $\begin{array}{l}\text { Organic B } \\
\text { Conventional }\end{array}$ & Foulum & 2008 & \\
\hline $\begin{array}{l}\text { Organic A } \\
\text { Organic B }\end{array}$ & & 2007 & 2 \\
\hline $\begin{array}{l}\text { Conventional } \\
\text { Organic A }\end{array}$ & Jyndevad & 2008 & \\
\hline Organic B & & 2007 & 2 \\
\hline
\end{tabular}

were ultrapure water containing $2 \mathrm{mM}$ sodium acetate (A) and $100 \%$ acetonitrile (B). $20 \mu \mathrm{L}$ was injected and a flow of $1 \mathrm{~mL} \mathrm{m^{-1 }}$ was maintained. The elution program was as follows: 0 to $45 \mathrm{~min}$. linear gradient $100 \% \mathrm{~A}$ to $85 \% \mathrm{~A} ; 45$ to 46 min. linear gradient $85 \%$ to $0 \% \mathrm{~A} ; 46$ to $50 \mathrm{~min}$, isocratic at $0 \% \mathrm{~A}$. Eluents were returned to the initial conditions within $2 \mathrm{~min}$. followed by $13 \mathrm{~min}$. equilibration. DAD spectra were collected in a range of 220 to $600 \mathrm{~nm}$. The equipment was operated by Chemstation for LC 3D systems Software (Agilent).

\subsubsection{LC- High Resolution Mass Spectrometry Analysis (LC-HRMS)}

The LC-HRMS system consisted of an Ultimate 3000 LC system coupled through a HESI II electrospray source to an Q-Exactive Orbitrap MS (Thermo Fisher Scientific, San Jose, CA, USA). The same column and gradient was used as described in section 2.2.2. The electrospray source was operated in negative ionization mode and the electrospray settings were as follows: electrospray voltage, $3.5 \mathrm{kV}$; sheath gas, 47.5 AU; auxiliary gas, 11.5 AU. The probe-heater was set to $410{ }^{\circ} \mathrm{C}$, and the heated capillary was set at $320^{\circ} \mathrm{C}$. Data were acquired by continuously alternating scan events: first a full-scan (135-1000 amu) followed by five all-ion fragmentation events $(\mathrm{m} / \mathrm{z}, 150 \pm 110 \mathrm{Da}, \mathrm{m} / \mathrm{z} 250 \pm 110$ $\mathrm{Da}, m / z, 350 \pm 110 \mathrm{Da}, m / z, 450 \pm 110 \mathrm{Da}$ and $\mathrm{m} / z 750 \pm 510 \mathrm{Da})$. The fragmentation normalized collision energy was stepped from 14 to 40 to $80 \mathrm{eV}$. The resolving power for the full-scan event was 70,000 and for the all-ion-fragmentation events this was 35,000 , defined at full width half maximum at $m / z, 200$. The other parameters for the mass spectrometer were automatically tuned with the tuning and calibration procedure. Before analysis, the mass calibration of the mass spectrometer was checked and re-calibrated if needed.

\subsubsection{Statistics}

Raw chromatographic data was baseline corrected (asymmetric least squares, $\lambda=10^{9}, \mathrm{p}=0.1$ ) and aligned for retention time correction by optimal reference sample selection using an in-house R-script ( $R$ version 3.1.2, The $\mathrm{R}$ Foundation, Vienna, Austria). Corrected and aligned data were subsequently subjected to Principal Component Analysis (PCA) to visualize the aligned chromatographic patterns using The Unscrambler X 10.3 package (Camo Software, Oslo, Norway). In order to determine significant relevance, the peak areas of the phenolic peak RT $=9.85$ min of organic and conventional samples were subjected to Analysis of Variance (ANOVA; Excel 2010, Microsoft, Redmond, WA, USA). All further calculations were performed in Excel 2010 (Microsoft).

\section{Results and Discussion}

\subsection{LC-DAD Analysis and Statistical Analysis of Phenolics}

All 36 samples were subjected to LCDAD analysis to assess both the bound and unbound phenolics. The chromatographic data were aligned. An example of an excerpt (2.0-20.0 min.) of such an array of chromatograms for unbound phenolics at $280 \mathrm{~nm}$ is shown in Fig. 1. The groups of chromatographic data of the bound and unbound phenolics measured at $280 \mathrm{~nm}$ and $320 \mathrm{~nm}$ were subsequently subjected to PCA.

The PCA on the unbound phenolics of individual measurements acquired at 280 $\mathrm{nm}$ revealed distinct clustering according to the production system (Fig. 2A). High positive sample scores on the first PC (explained variance $=93 \%$ ) were associated with conventional production, whereas high negative scores were associated with organic samples. No consistent differences between the Organic A and B group samples were observed. Furthermore, some cluster- 


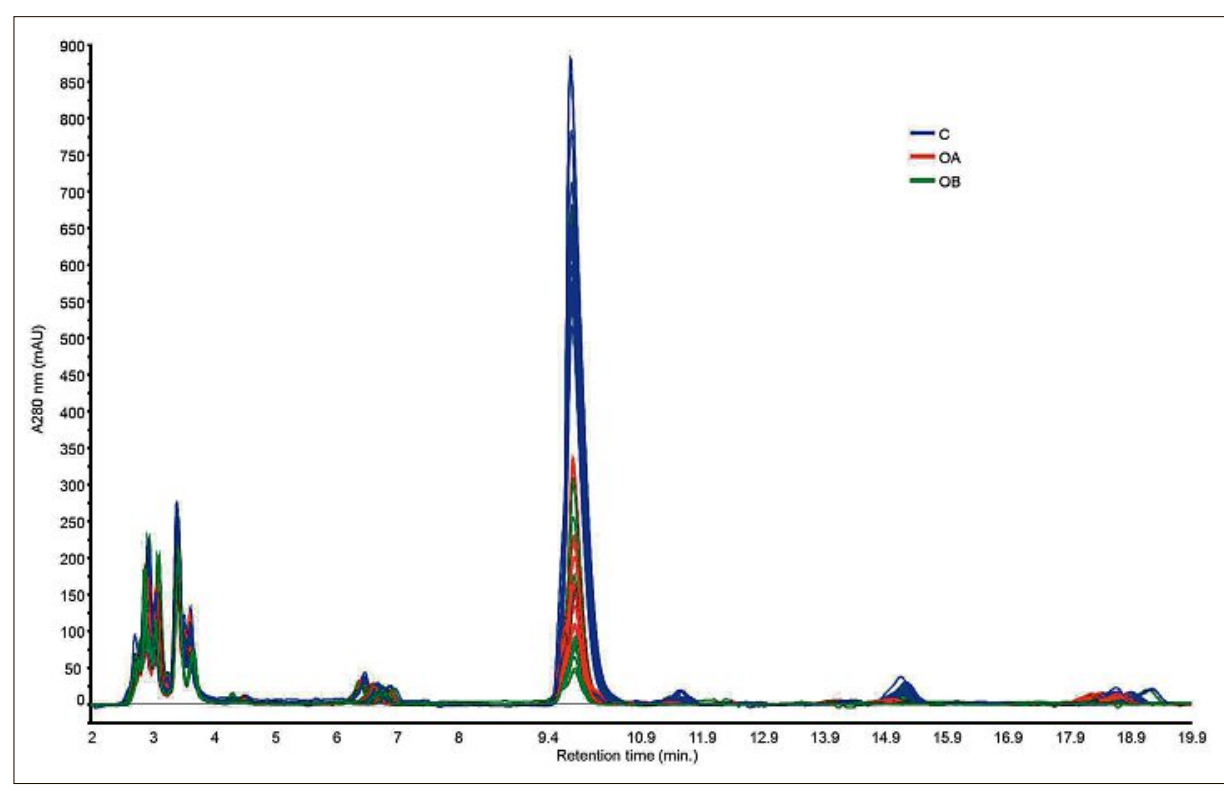

Fig. 1. Overlay of chromatograms of unbound phenolics at $280 \mathrm{~nm} . \mathrm{C}=$ conventional, $\mathrm{OA}=$ organic $\mathrm{A}, \mathrm{OB}=$ organic $\mathrm{B}$.

ing was observed in the second dimension $(\mathrm{PC} 2$ explained variance $=4 \%)$. This clustering was due to the year-to-year variation of the wheat samples. The production location did not reveal any consistent clustering in the first two dimensions of the PCA plot. All other combinations of free and unbound phenolics at 280 and $320 \mathrm{~nm}$ were subjected to PCA and examined, however no clustering within growing systems was observed upon PCA analysis.

For identification of the set of phenolics that allowed the separation between the organic and conventional growing systems, the loadings plot of the PCA of the unbound phenolics measured at $280 \mathrm{~nm}$ was examined (Fig. 2B). The loadings plot was dominated by one chromatographic peak with a maximum at $9.85 \mathrm{~min}$, flanked by several minor phytochemical compounds at retention times 2.50 to $3.85 \mathrm{~min}$, $6.51 \mathrm{~min}, 11.69 \mathrm{~min}$, and $15.35 \mathrm{~min}$.

The predominant peak at $9.85 \mathrm{~min}$ observed in the loadings plot (Fig. 2B) was quite surprising and would indicate that this peak allows separation of the organic and conventional wheat on its own. It is very uncommon that organic products can be distinguished from their conventional counterparts by a single marker. ${ }^{[4,9,10]}$ Instead of using data-points of aligned full LC chromatograms, individual peak areas could be used and reduce the complexity of both the analysis and data processing. Therefore the peak areas were compared (Fig. 3). Significantly different areas of this particular peak (ANOVA, $\mathrm{P}<0.05$ ) for organic and conventional wheat samples were observed. From the phenolic compound analyzed, the results allow for consistent discrimination between organic and conventional wheat production systems for these Danish wheat samples.
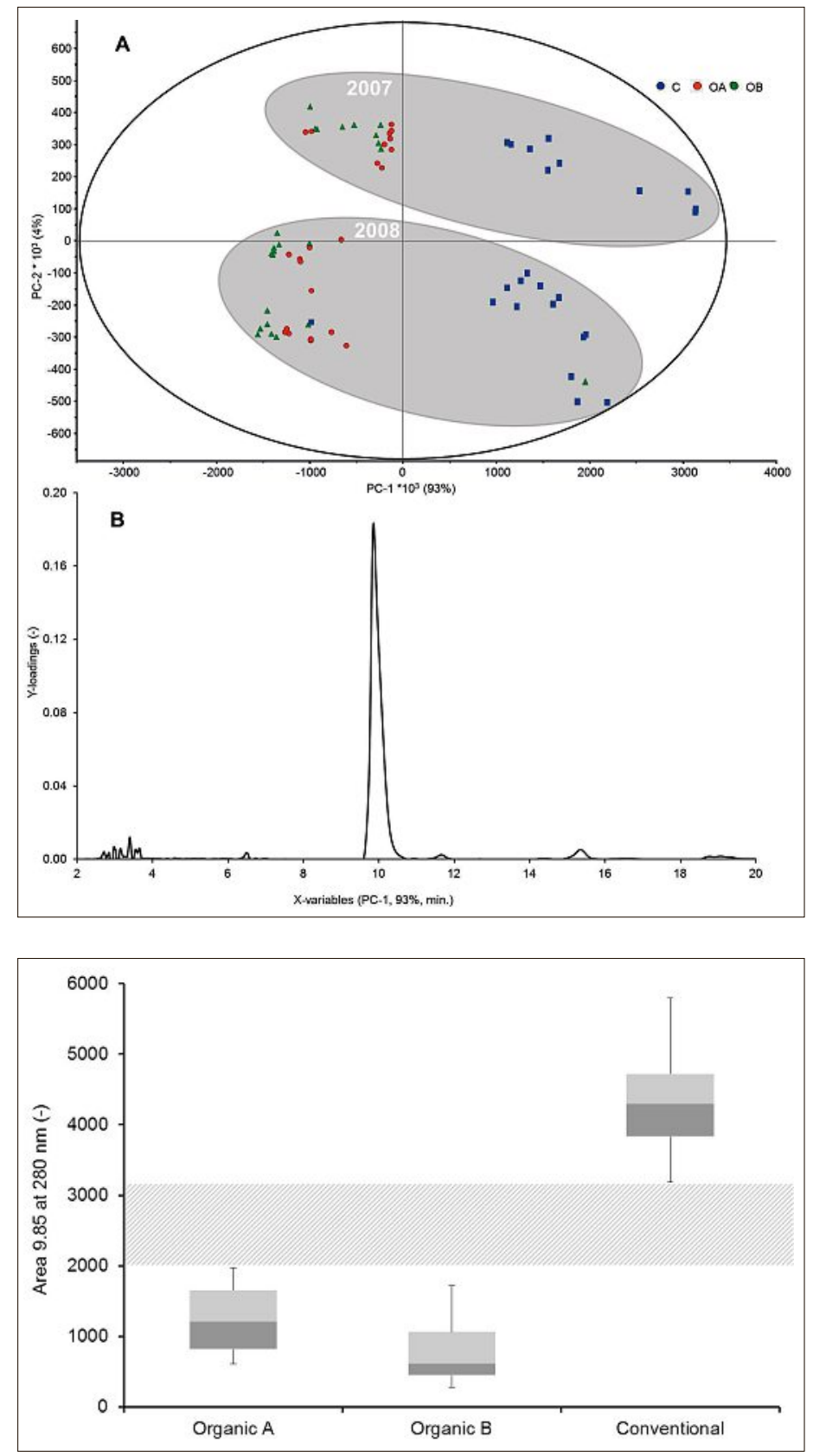

\subsection{Identification of the Single Marker}

The identification of the single marker that was discerned in section 3.1 was performed by mass-spectrometric analyses. The parent mass $[\mathrm{M}-\mathrm{H}]^{-}$determined was $\mathrm{m} / \mathrm{z}$ 153.02, which indicates the presence of the dihydroxybenzoic acid: protocatechuic acid (Fig. 4A). Upon examination of the $\mathrm{MS}^{2}$ spectrum generated by high energy collision induced dissociation (HCD), typical fragments around $m / z, 110$ were found which could be associated with ejection of the carboxylic acid group [M-H$\mathrm{CH}_{2} \mathrm{O}^{-}$(Fig. 4B). Multiple possibilities for this ejection occurred resulting in $\mathrm{m} / \mathrm{z}$ 109.03, 110.03 and 111.03 fragments. The fragment at $\mathrm{m} / \mathrm{z}$ 59.01 could be a result of a benzene ring fragmentation which is also observed with, for example, dihydrocaffeic acid. ${ }^{[11]}$ Due to the higher fragmentation energies used with $\mathrm{HCD}$, benzene ring
Fig. 2. A: Plot of the first two dimensions of the PCA of LC data points after base-line correction and alignment of unbound phenolics at $280 \mathrm{~nm}$ for retention time window of 2.0 to 20.0 min. $C=$ conventional, $\mathrm{OA}=$ organic $A, O B=$ organic B. B. Associated loadings plot of PC1.
Fig. 3. Boxplot of the areas underneath phenolic compound (RT $=9.85 \mathrm{~min}$ ) for organic $A$ and $B$ and conventional wheat. 


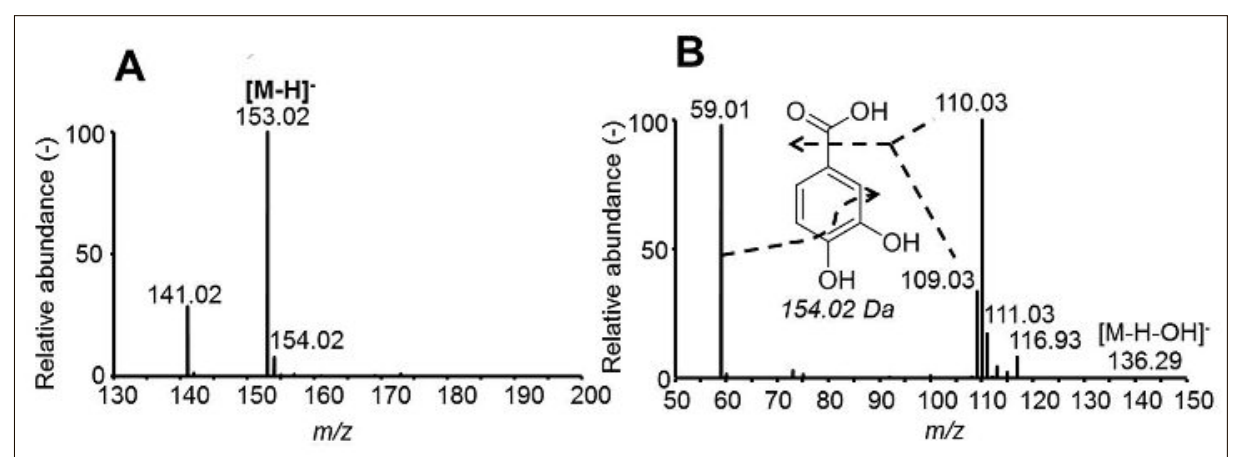

Fig. 4. A. Full scan negative mode MS spectrum of protocatechuic acid as found in the wheat extracts at RT $9.85 \mathrm{~min}$. B. $\mathrm{MS}^{2}$ hcd fragment spectrum of $\mathrm{m} / \mathrm{z} 153.02$ and correlation of the main fragments with the protocatechuic acid molecular structure.

fragmentation may occur, although this type of fragmentation is unusual in negative mode. Finally, loss of $\mathrm{OH}$ groups was observed at $m / z, 136.29$. From the information gathered from the elemental composition and fragmentation pattern, we expect that the phenolic compound which allows differentiation between the organic and conventional wheat samples is protocatechuic acid.

The tentative annotation of protocatechuic acid as the single marker for authentication of organic grown wheat is remarkable for three reasons. Firstly, protocatechuic acid was not reported to be a highly occurring phenolic in wheat, in contrast to ferulic acid, $p$-coumaric acid, syringic acid, vanillic acid and caffeic acid. ${ }^{[7]}$ Either protocatechuic acid was systematically overlooked or, although unlikely due to the sample set-up, it was specifically overproduced by this wheat variety. Secondly, the significant differences in protocatechuic acid levels may be due to external factors which (partly) activated or blocked a specific part of the benzoic acid pathway. Protocatechuic acid is biosynthesized via 3-dehydroshikimic acid in the shikimate pathway in plants. One of the precursors of 3-dehydroshikimic acid is D-arabinoheptulosonic acid 7-phosphate of which the enzymatic biosynthesis was reported to be susceptible to specific (de)activation by environmental stimuli. ${ }^{[12]}$ Although this does not explain why protocatechuic acid is specifically upregulated in the conventional production system, environmental factors could result in increase or decrease of dihydroxybenzoic acid production as observed for protocatechuic acid. Thirdly, in order to differentiate between growing systems, often a group of markers is required, as the growing process is often influencing many parts of the biosynthetic pathway. In this unique case, the organic production system led to almost specific down-regulation of the protocatechuic acid production. Phenolic compounds are secondary metabolites that are widely distributed in plant foods such as fruits and vegetables but also in cereals, legumes, tea, coffee and chocolate. Their contents in organic products and their conventional counterparts have been extensively investigated. Usually higher concentrations of phenolics have been reported in organic plant products. ${ }^{[13,14]}$ The different phenolic content in organically grown crops has been ascribed to the absence of synthetic pesticides in organic farming, resulting in higher exposure to stressful situations, which would lead plants to produce secondary metabolites as a defensive mechanism. ${ }^{[15,16]}$ The availability of inorganic nitrogen, which depends on the type of fertilizer, can also modulate plant biosynthetic pathways, resulting in more limited formation of phenolic compounds in organic crops.[17] It was shown for this specific sample set that the nitrogen yield of the wheat on dry matter was systematically higher for the conventional system than for both organic systems. ${ }^{[8]}$ The latter may explain the different levels observed in the organic and conventional wheat samples in the current study.

\section{Conclusions}

Organic and conventional wheat proharvested over a two-year period demonstrated significantly different levels of protocatechuic acid. It is therefore considered a promising marker that can be determined by LC analysis of unbound phenolics at $280 \mathrm{~nm}$. In the current study, a well-defined sample set of Danish origin was used. The extension to wheat of other provenance as well as commercial samples would need further research. duced in three locations in Denmark and

\section{Acknowledgements}

The authors acknowledge the financial support provided by the CORE Organic II Funding Bodies, being partners of the FP7 ERA-NET Project, CORE Organic II (Coordination of European Transnational Research in Organic Food and Farming systems, Project No. 249667) via the AuthenticFood Project. Furthermore, the authors would like to acknowledge the funding of the research project from the Ministry of Economic Affairs in Netherlands, as well as through the Wageningen UR KBproject Authenticity-traceability. The authors gratefully acknowledge the EU CORE Organic II project partners for providing the Danish wheat sample material: Jørgen E. Olesen and co-workers via the CropSys project (Aarhus University), as well as Kristian Holst Laursen and Søren Husted (University of Copenhagen).

Received: December 10, 2015

[1] S.R. Chapman, L.P. Carter, 'Crop Production: Principles and Practices', W.H. Freeman and Co. San Francisco, CA, USA, 1976.

[2] FAOSTAT, http://faostat3.fao.org/browse/ rankings/commodities_by_regions/E, accessed Nov. 29, 2015

[3] EUR-LEX, http://eur-lex.europa.eu/legalcontent/EN/TXT/?uri=OJ:L:2008:250:TOC accessed Dec. 3, 2015.

[4] E. Capuano, R. Boerrigter-Eenling, G. van der Veer, S. M. van Ruth, J. Sci. Food Agric. 2012, 93, 12 .

[5] K. K. Adom, R. H. Liu, J. Agric. Food Chem. 2002, 50, 6182 .

[6] A. S. Hole, S. Grimmer, M. R. Jensen, S. Sahlstrøm, Food Chem. 2012, 133, 969.

[7] N. Okarter, C.-S. Liu, M. E. Sorrells, R. H. Liu, Food Chem. 2010, 119, 249.

[8] K. H. Laursen, J. K. Schjoerring, J. E. Olesen, M. Askegaard, U. Halekoh, S. Husted, J. Agric. Food Chem. 2011, 59, 4385.

[9] K. H. Laursen, J. K. Schjoerring, S. D. Kelly, S Husted, Trends Anal. Chem. 2014, 59, 73.

[10] I. Reinholds, V. Bartkevics, I. C. J. Silvis, S. M van Ruth, S. Esslinger, J. Food Compos. Anal. $\mathbf{2 0 1 5}, 44,56$

[11] MassBank, http://www.massbank.jp/jsp/ Dispatcher.jsp $?$ type $=\operatorname{disp} \& i d=K O 000588 \&$ si te $=0$, accessed Nov. 27, 2015.

[12] D. Vauzour, K. Vafeiadou, J. P. E. Spencer, in 'Phytonutrients', Eds. A. Salter, H. Wiseman, G. Tucker, Blackwell Publishing Ltd, Chickhester, UK, 2012, p. 115.

[13] G. P. P Lima, S. Rocha, M. Takaki, P. R. R. Ramos, E. O. Ono, Int. J. Food Sci. Technol. 2008, 43, 1838.

[14] D. K. Asami, Y. J. Hong, D. M. Barrett, A. E Mitchell, J. Agric. Food Chem. 2003, 51, 1237.

[15] C. K. Winter, S. F. Davies, J. Food Sci. 2006, 71, 117.

[16] K. Woese, D. Lange, C. Boess, K. W. Bogl, J. Sci. Food Agric. 1997, 74, 281.

[17] O. Daniel, M. S. Meier, J. Schlatter, P. Frischknecht, Environ. Health Perspect. 1999. 107, 109. 